Images dans le monde ibérique et ibéricoaméricain

9 | 2016

Innovations politiques et culturelles dans les pays andins

\title{
Regards, miroirs et faux-semblants dans Zona sur de Juan Carlos Valdivia
}

\section{Erich Fisbach}

\section{(2) OpenEdition \\ Journals}

\section{Édition électronique}

URL : https://journals.openedition.org/agedor/1211

DOI : 10.4000 /agedor.1211

ISSN : 2104-3353

\section{Éditeur}

Laboratoire LISAA

Référence électronique

Erich Fisbach, « Regards, miroirs et faux-semblants dans Zona sur de Juan Carlos Valdivia », L'Âge d'or [En ligne], 9 | 2016, mis en ligne le 01 mars 2016, consulté le 21 septembre 2021. URL : http:// journals.openedition.org/agedor/1211; DOI : https://doi.org/10.4000/agedor.1211

Ce document a été généré automatiquement le 21 septembre 2021.

L'Âge d'or. Images dans le monde ibérique et ibéricoaméricain 


\title{
Regards, miroirs et faux-semblants dans Zona sur de Juan Carlos Valdivia
}

\author{
Erich Fisbach
}

1 Depuis la fin du XXe siècle, la Bolivie connaît de profonds bouleversements politiques et un rééquilibrage social avec l'arrivée d'Evo Morales à la présidence de la république en 2005. La géographie de la ville de La Paz était le reflet de ce déséquilibre, les plus pauvres, les Indiens, occupant les hauteurs de la ville alors que les classes aisées avaient investi les zones de moindre altitude. C'est ainsi qu'est né, dans les années 1950, le quartier de Calacoto, situé à environ 3300 mètres d'altitude, alors que le centre se trouve lui à 3600 mètres et l'ancien quartier populaire devenu une véritable ville ces dernières années, $\mathrm{El}$ Alto, surplombe la ville de La Paz à plus de 4000 mètres d'altitude. Cette zone des beaux quartiers s'est considérablement étendue et métissée depuis quelques années et on la désigne désormais sous le nom de «Zona sur ». Tel est le titre du quatrième film de Juan Carlos Valdivia, dont le premier long-métrage, Jonás y la ballena rosada (1995), adaptation d'un roman de Wolfango Montes Vanucci, faisait le portrait d'une société en crise et en proie aux trafiquants de drogue, alors que le deuxième, American visa (2005), racontait les aventures d'un professeur d'anglais qui tente d'obtenir un visa pour les États-Unis, et qui se heurte à toute sorte de mésaventures.

2 Zona sur, tourné avec une caméra numérique mais distribué en $35 \mathrm{~mm}$, et sorti sur les écrans en 2009, est donc le troisième film de Juan Carlos Valdivia. Il diffère totalement des précédents sur tous les plans, à commencer par le fait qu'il s'agit cette fois d'un scénario original alors que les deux premiers films sont des adaptations de romans à succès ${ }^{1}$, mais aussi et surtout du point de vue esthétique. En effet, le récit conventionnel et le montage somme toute classique des deux premiers films cèdent ici la place à une mise en scène à la fois très personnelle - ce que Juan Carlos Valdivia revendique dans toutes les interviews qu'il a accordées - et complexe, qui se caractérise en particulier par l'utilisation d'une caméra en constant déplacement et par un montage qui prolonge ce mouvement et donne au film une grande fluidité qui n'est pas sans surprendre le spectateur. Zona sur dresse le 
portrait d'une famille qui appartient à cette classe aisée qui vit dans ces quartiers résidentiels, mais une classe aisée en perte de vitesse et en perte de repères. Dans le portrait qui est dressé de cette famille, on remarque d'emblée l'absence du père, l'absence d'une figure paternelle que n'incarnent ni Wilson, le cuisinier et employé de maison ${ }^{2}$, ni Patricio, le fils aîné, décrit comme un adolescent irresponsable, ni Andrés, le plus jeune fils. L'histoire de cette famille se déroule ainsi à un moment de grands changements dans cette société bolivienne, comme le souligne Juan Carlos Valdivia dans plusieurs interviews et comme le rappelle par exemple Juan José Olivares dans la rubrique Espectáculos du journal en ligne La Jornada :

Se trata de un "retrato de la clase alta paceña", con el que el realizador Juan Carlos Valdivia pretende mostrar a una familia en un momento en que Bolivia ha elegido efectuar grandes cambios sociales ${ }^{3}$.

3 En l'absence du père, cette famille se compose dès lors de la mère, Carola, de ses trois enfants, les deux adolescents, Patricio et Bernarda, et le jeune Andrés. À ce noyau familial s'ajoutent les deux employés aymaras, Wilson et Marcelina. Quelques autres personnages rejoignent ce cercle familial, parmi lesquels Carolina, la petite amie de Patricio ; Erika, la petite amie de Bernarda ; la tante Rosario ; les amis de Bernarda et Patricio, ou encore les comadres de Carola que l'on retrouve dans les dernières séquences du film, en particulier la femme indienne qui lui propose de lui acheter sa maison cash. En dehors de quelques très rares séquences filmées hors de la maison, dont une sur l'Altiplano, précédée de quelques scènes de rue, l'essentiel du film est tourné dans cette maison et dans les différents espaces qui la constituent, y compris le toit ou le jardin. Zona sur décrit dès lors une sorte de huis clos qui ne raconte pas réellement une histoire avec un début, un développement et un dénouement classiques, mais plutôt une série de moments qui constituent le présent, le quotidien des membres de cette famille de jailones, pour reprendre ce bolivianisme qui désigne la haute société et que l'écrivain bolivien Edmundo Paz Soldán définit comme "... alguien de la High society, y la connotación suele ser negativa"4, un quotidien dans lequel les signes d'une décadence sourde et inexorable se multiplient.

4 La caméra de Juan Carlos Valdivia circule ainsi dans un espace, dans des espaces, qu'elle contribue à modeler, elle tourne parfois sur elle-même, parfois autour des personnages ou encore au-dessus d'eux, sans jamais s'attarder sur eux, comme s'il ne s'agissait pas de les suivre, mais plutôt de s'attacher aux lieux auxquels ils s'identifient, comme la cuisine pour Wilson, l'homme à tout faire de la maison, la chambre ou la salle de bains de la mère, Carola, ou encore la chambre du fils aîné, Patricio, etc. Cette structure du film, composé exclusivement de plans-séquences qui nous renvoient chacun à un lieu, a obligé Juan Carlos Valdivia et son équipe à résoudre certains impératifs techniques et à adopter des dispositifs qui rendent possible cette fluidité, ce constant mouvement de l'image, et permettent les déplacements de la caméra sur son axe - soit les panoramiques circulaires - ou autour du sujet filmé - soit les travellings circulaires. Le réalisateur a également eu recours à un logiciel de modélisation en 3D, sketch up, afin de visualiser l'espace, d'anticiper la perception du spectateur d'imaginer les mouvements de caméra, laquelle tourne le plus souvent sur elle-même dans le sens des aiguilles d'une montre.

5 Juan Carlos Valdivia justifie la forme particulière qu'il donne à son film en expliquant qu'il voulait rompre avec le langage dialectique qui construit le récit par la juxtaposition de plans :

Quería romper con el lenguaje dialéctico del cine que narra con la yuxtaposición de imágenes o que a veces tan solo se limita a cubrir acciones con una variedad de 
ángulos. Tampoco quería seguir a los personajes e imponer el plano secuencia en la

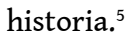

6 Une autre explication à ces choix de mise en scène se trouve dans les théories du philosophe allemand Peter Sloterdijk, auteur de la trilogie Sphères, qui se décline en trois volumes, Bulles, Globes, Écumes ${ }^{6}$. Ainsi, Juan Carlos Valdivia a voulu refléter dans son film ces différentes bulles dans lesquelles sont enfermés les personnages, bulles qui les protègent mais qui les isolent à la fois dans l'incompréhension et l'égoïsme individuel et social. Juan Carlos Valdivia reprend ainsi à son compte les théories de Peter Sloterdijk, les mouvements circulaires de la caméra matérialisant en quelque sorte ces bulles et rendant ce huis clos oppressant. Comme l'explique Valdivia :

La experiencia del espacio siempre es la experiencia primaria de existir. Vivimos en esferas, en atmósferas, desde la primera en el útero de la madre. Pero también existen las esferas sociales y la espuma, que es el siempre cambiante conglomerado de esferas individuales vinculadas entre sí. Sloterdijk es el filósofo de las vivencias; usa mucho el arte para explicar e ilustrar sus teorías. ${ }^{7}$

7 De cette façon, si les personnages sont enfermés dans des préjugés, des habitudes, des bulles sociales, culturelles, s'ils sont enfermés dans ce huis clos que constituent la maison et ses différents micro-espaces et dans cet espace que tracent et que délimitent les mouvements de caméra, en même temps, cette circularité des plans n'est pas englobante. En effet, l'image est fuyante et le cadre ne laisse souvent voir que des fragments de l'espace, des moitiés de visages, des fragments des corps des personnages qui disparaissent rapidement dans le hors champ à mesure que la caméra tourne sur ellemême. Cette dialectique de circularité et de fragmentation enferme en quelque sorte contradictoirement les personnages dans un environnement étouffant. Dans un même temps, les regards, les images se démultiplient par la prolifération de miroirs, de fenêtres, etc., qui ne reflètent pas le réel mais proposent la vision d'une réalité bouleversée, également fragmentée et éclatée, et contribuent à souligner, voire à symboliser le caractère insaisissable d'un monde qui échappe aux personnages.

8 Zona sur se compose dès lors de seulement quatre-vingt-sept plans, qui sont par ailleurs tous des plans-séquences de durées variables et qui se caractérisent par les mouvements essentiellement circulaires de la caméra qui tourne généralement sur elle-même, le plus souvent de gauche à droite, et qui ne suit pas nécessairement les actions des personnages qui se retrouvent hors champ lorsqu'ils ne suivent pas eux-mêmes le sens ou l'axe de déplacement latéral de la caméra. Si l'on pense à la définition de l'espace au cinéma relevée par Eric Rohmer ${ }^{8}$, soit, l'espace pictural - l'image projetée sur l'écran et perçue comme une représentation plus au moins exacte du monde extérieur -, l'espace architectural - l'existence objective de ces parties du monde fabriquée -, et l'espace filmique - soit l'espace virtuel reconstitué pour le spectateur par des éléments fragmentaires donnés par le film -, alors cette représentation de l'espace est censée donner des informations sur le lieu où se déroule l'action du film et sur l'action à proprement parlé, soit ce qui se passe à l'intérieur du plan. On voit bien selon cette définition que si les techniques cinématographiques visent à faire oublier la caméra et à placer l'action au cœur du dispositif narratif, il n'en est rien dans Zona sur où la caméra ne se contente pas de montrer et de suivre l'action des personnages mais construit bien au contraire un espace qui enferme ces personnages dans cet espace labyrinthique de la maison tout en les sortant du champ.

9 Le premier plan-séquence du film, d'une durée de 1'26" est emblématique de l'esthétique $\mathrm{du}$ film. En effet, après un générique en lettres blanches sur fond noir et une bande 
sonore qui fait entendre un son électronique filé, celui-ci cède la place au bruit de moteur d'une voiture qui se rapproche, alors que l'image en mouvement qui apparaît est celle d'une grille qui donne sur la rue. La caméra glisse ensuite sur un miroir dans lequel on voit la voiture qui s'apprête à pénétrer dans le jardin, puis une indienne qui referme la grille et dont on ne voit ensuite que le haut du crâne alors qu'elle se dirige vraisemblablement vers l'entrée de la maison. La caméra glisse ensuite sur une haie intérieure épaisse qui ne permet pas de voir ce qu'il y a de l'autre côté, alors que l'on entend la voix d'un homme qui interpelle la femme en aymara; celle-ci lui répond en aymara également alors que la caméra arrive sur les deux personnages et que nous découvrons le petit garçon que l'homme a sans doute ramené du club de tennis. L'échange se poursuit en espagnol avec le petit garçon qui entre dans la maison avec cet homme, Wilson, alors que la femme, Marcelina, reste dans le jardin et que la caméra poursuit son mouvement circulaire pour revenir à son point de départ, c'est-à-dire la grille que Marcelina referme cette fois, puis sur le miroir dans lequel on la voit s'éloigner. À l'image de cette grille que referme Marcelina, cette première séquence nous introduit d'emblée dans un univers fermé, dont nous voyons bien ici qu'il est à la fois un refuge, mais aussi une sorte de lieu de réclusion séparé du monde par une grille et une chaîne fermée avec un cadenas.

Dès cette première séquence, nous voyons que la caméra ne calque pas ses mouvements sur ceux des personnages, qu'elle ne suit pas leurs déplacements et leurs actions ; en effet cette caméra très mobile, mais dont les mouvements sont à la fois lents empreints d'une certaine sérénité, donne plutôt l'impression de montrer l'espace dans lequel se déroule une action que nous devinons par la bande sonore et que ne nous voyons que partiellement, soit lorsque l'image ne cadre que fragmentairement un personnage, comme c'est le cas dans cette première séquence lorsque nous voyons le haut du crâne de Marcelina passer dans le champ, soit lorsque l'image se reflète dans un miroir - comme cela se reproduit dans de nombreuses séquences du film... Ajoutons dans cette première séquence la grille qui ne laisse voir qu'une partie de l'espace extérieur, lequel n'est par ailleurs visible que dans de très rares séquences, comme celles dans lesquelles Andrés est sur le toit de la maison et parle à ce personnage fictif qu'il nomme Spielberg (plans $\mathrm{n}^{\circ} 12$, $19,40,82)$, ou lorsque la caméra vole littéralement, comme un cerf-volant, au-dessus de la maison et du jardin dans la dernière séquence du film. Cet espace extérieur est également visible lors d'une séquence composée de cinq plans au cours de laquelle Wilson, passant outre l'interdiction de Carola, prend la voiture pour assister à une cérémonie pour l'enterrement de son fils. Cet espace extérieur est néanmoins vu depuis l'intérieur de la voiture et à travers le pare-brise ruisselant de pluie. Si, au cours de ce plan, la pluie brouille l'image de la ville de La Paz que Wilson et Andrés - qui s'est introduit clandestinement dans la voiture - sont en train de quitter, brouillage accentué par le fait que les essuie-glaces ne fonctionnent pas, le rétroviseur renvoie quant à lui l'image d'Andrés. Plus loin, alors qu'Andrés est resté dans la voiture et regarde vers l'extérieur, Wilson et trois autres personnes mettent un petit cercueil blanc dans la voiture et nous devinons que l'homme et le petit garçon se trouvent dans le quartier de El Alto. Puis, après une discussion à travers la vitre baissée de la voiture avec un policier qui lui réclame ses papiers avant de le laisser continuer, la caméra, placée au bord de la route, se déplace vers la droite en un court travelling pour suivre la voiture qui passe à côté d'un cimetière. Dans le dernier plan de cette séquence, nous assistons à une cérémonie traditionnelle; le mouvement circulaire de la caméra symbolise ici la communion et le 
partage entre ces hommes et ces femmes avant de s'élever et de nous laisser voir le lac Titikaka qui ne reflète que les nuages gris.

11 Cette séquence, constituée de cinq plans et qui ne dure que trois minutes et demie, est bien la seule séquence du film qui se situe en dehors de l'espace délimité, sinon fermé, de la maison et du jardin et donc la seule qui se situe en dehors de la Zona sur, que nous ne voyons par ailleurs pratiquement jamais, en dehors des images vues du toit et que la caméra y accompagne Andrés. Ce regard sur cet autre espace, de cette autre réalité, dont on ne perçoit que quelques images fragmentaires à travers les vitres embuées de la voiture, à l'image des maisons en adobe de El Alto ou du slogan à la gloire d'Evo Morales, n'est rendu possible que par la présence d'Andrés qui fait le lien entre ces deux mondes. Andrés est en effet celui qui, avec son ingénuité d'enfant, pose des questions, à Wilson, à Marcelina, aux vendeurs de pommes de terre, mais aussi à sa mère, comme à la fin de cette séquence lorsqu'il demande à Wilson de quoi est mort son fils, sans obtenir de réponse. Paradoxalement, Andrés, le petit garçon, est ainsi celui qui donne à la caméra l'occasion de s'évader de l'espace fermé de la maison et d'apercevoir l'espace extérieur, comme c'est le cas dans cette séquence de la sortie qu'il fait en compagnie de Wilson, ou encore lorsque cette caméra l'accompagne sur le toit de la maison nous permettant de voir au loin les toits des maisons voisines ou les montagnes qui entourent cette zone, parmi lesquelles on distingue l'éminence que l'on connait sous le nom de Dent du diable.

Si la maison est une sorte de bulle protectrice, elle se révèle être aussi en quelque sorte comme un espace castrateur qui symbolise l'enfermement d'une classe sociale dans un modèle qui s'autoalimente, comme l'explique Erika, la petite amie de Bernarda à Carolina, qui est quant à elle la petite amie de Patricio, qui lui confesse qu'elle rêve de partir loin de ce milieu, loin de sa future belle-mère, en lui disant qu'elle ne fera que reproduire le modèle du matriarcat bolivien, parce que c'est là le rôle que toutes les femmes vont jouer à un moment ou à un autre, avant de l'appeler "futura Carola» (plan n' 36, 47'34"). Bernarda menace pour sa part sa mère d'aller vivre chez Erika, dans le quartier plus populaire de Miraflores et, plus tard, la tante Rosario se demande pourquoi ils ne sont pas encore partis de Bolivie dans la mesure où il n'y a aucun avenir et que Patricio s'apprête à partir étudier en Espagne. Lors de cette même réunion de famille, Carola affirme qu'elle ne rêve que de les voir partir pour se retrouver enfin seule (séquence $n^{\circ} 44$ ). Andrés quant à lui s'évade sur le toit, dans la petite cabane en bois construite dans un arbre, ou encore dans ses rêves.

La maison est ainsi un espace ambivalent, à la fois protecteur et castrateur dans lequel on retrouve plusieurs micro-espaces plus spécifiquement associés à l'un ou l'autre des personnages. La caméra parcourt différentes pièces de la maison que l'on associe plus spécifiquement à certains personnages, comme la cuisine à Wilson ou encore le jardin à Marcelina ${ }^{9}$. Nous retrouvons par ailleurs la chambre de Bernarda, celle de Patricio, dans laquelle la salle de bains n'est séparée de la chambre que par une cloison en verre et dans laquelle lui et Carolina se livrent à des ébats amoureux qu'ils filment à l'aide d'une caméra vidéo placée devant le lit; la chambre de Carola, ainsi que sa salle de bains, ou encore la chambre de Wilson qui semble se réduire à un petit lit et à une armoire. D'autres espaces sont plutôt des espaces partagés, comme c'est le cas notamment de la salle à manger qui est pratiquement la seule pièce dans laquelle se retrouvent la mère et ses trois enfants, voire tous les personnages. À l'inverse de la salle à manger, le salon n'est pas véritablement un espace de partage et de sociabilité et apparaît plutôt comme un lieu de pouvoir, abandonné en quelque sorte par ce pouvoir. Deux plans sont à cet égard tout 
à fait significatifs; le premier (plan $n^{\circ} 23$ ) se situe à la $24^{\mathrm{e}}$ minute du film et il s'agit du premier plan tourné dans ce salon. On y voit Wilson et Marcelina qui discutent en aymara et s'approprient l'imposant canapé blanc qui occupe le centre du salon, alors que la caméra qui se déplace de gauche à droite démultiplie l'image qui se reflète dans un grand miroir. À la fin du film, nous retrouvons ce même salon occupé cette fois par Carola et ses comadres (plan $\left.n^{\circ} 79,94^{\prime} 50^{\prime \prime}\right)$; le canapé est occupé cette fois par les deux femmes indiennes qui viennent par ailleurs proposer à Carola de lui acheter sa maison cash pour la démolir et construire un immeuble à la place. Ainsi, le salon, loin de représenter le pouvoir d'une classe sociale dominante devient ainsi le lieu symbolique de la chute de cette même classe sociale, à l'image de ce canapé blanc occupé par des Indiens, à l'image aussi de ces gros coquillages qui symbolisent quant à eux le rêve de la mer perdue.

Il est intéressant de souligner que les nombreux espaces intérieurs de cette maison, dans laquelle on a l'impression que la seule porte fermée est celle de la chambre de Wilson sur laquelle Carola n'hésite pas à tambouriner lorsqu'elle a besoin de lui, sont reliés entre eux par des couloirs, des escaliers sur lesquels la caméra glisse indéfectiblement. En dépit de cela, cet espace intérieur donne l'impression d'être labyrinthique ; cette impression est accrue par les incessants mouvements de la caméra qui tourne sur son axe et par un cadre qui se démultiplie sans cesse, en se projetant notamment dans les miroirs, ou en se déformant dans les vitres et qui ne s'attache pas à intégrer entièrement les personnages. Mentionnons à cet égard le $5^{\mathrm{e}}$ plan du film, qui illustre parfaitement cette construction fragmentaire de l'espace. Cette scène se situe dans la salle de bains de Carola, alors que celle-ci se prépare à sortir et que Wilson l'aide à se préparer. Nous voyons ici au début du plan que Carola est en train d'uriner, mais que la porte de la salle de bains est cependant ouverte et que Wilson attend à l'extérieur; la caméra se déplace ici aussi de gauche à droite à l'espace tout comme les mouvements des personnages sont révélés par les reflets dans les multiples miroirs, sans que nous n'ayons cependant une idée très exacte ni de la forme, ni des dimensions de cette salle de bains (plan $n^{\circ} 5$ ).

À propos de l'importance des miroirs dans Zona sur, Sebastián Jorge Morales Escoffier affirme dans le mémoire de fin de diplôme qu'il a consacré à ce film que

la intención de hacer de la casa un espejo de los espectadores de la sala, es sin duda consciente en Valdivia, así en la entrevista realizada para esta investigación, el director del film afirmaba contundentemente que: "Zona sur te pone un espejo y por eso a mucha gente no le gusta, porque a la gente no le gusta mirarse en los espejos". ${ }^{10}$

Dans un article au titre significatif, «Zona sur : ver y no mostrar », Mary Carmen Molina écrit quant à elle que :

La casa llena de espejos donde mirarse una y otra vez, cuidadosamente tupida de copas y frascos de cristal a través de los que la cámara cruza con los personajes, la casa donde las ventanas se cierran y los ojos se vuelven sobre sí mismos. Es a través de estas insistencias que se configura una de las intenciones de esta película: retratar a esta familia de la Zona Sur paceña no pasa por el hecho de ofrecer el reflejo de una realidad sino la construcción de una versión de ésta, una mirada profundamente personal donde no se cede ante el estereotipo y donde enfrentarse al espejo es también traspasarlo. ${ }^{11}$

Cette construction particulière contribue à donner une impression d'étouffement, de huis clos, en dépit du fait que toutes les portes restent ouvertes et, hormis le premier plan, l'on ne voit jamais les personnages rentrer ni sortir de la maison comme si aucun chemin ne conduisait vers la sortie, ce qui correspond par ailleurs à l'intention du réalisateur : 
Queríamos que la cámara corte como un cuchillo, ver siempre las partes en lugar de verlo todo, encuadres en los que se cortaran los rostros y partes de los cuerpos de los personajes. Queríamos también ver a través de algo, detrás de algo, crear la sensación de encierro, claustrofobia, asfixia.

La cámara que gira constantemente no sólo hace referencia a las esferas y al tiempo, sino que construye un laberinto donde todos los cuartos se conectan entre sí, pero ninguno conduce a la salida. ${ }^{12}$

Cette impression d'enfermement est suggérée à plusieurs reprises dans le film où nous retrouvons plusieurs plans dans lesquels les mouvements de caméra sont différents, celleci ne tournant plus sur elle-même, et dans lesquels cette caméra est située à l'extérieur alors que les personnages sont à l'intérieur et regardent vers l'extérieur ${ }^{13}$. Le $63^{\mathrm{e}}$ plan est à cet égard très symbolique; la caméra se déplace en effet de gauche à droite et effectue un travelling arrière qui commence sur Andrés que l'on voit assis sur le toit de dos, et ensuite la caméra montre les différents personnages regardant par des fenêtres différentes, Patricio seul, puis Carola et Wilson et enfin Bernarda et Marcelina avant que la caméra ne s'arrête sur la famille éclatée.

Depuis son premier film, Jonas y la ballena rosada, Juan Carlos Valdivia explore la réalité bolivienne, celle de la crise économique des années 80/90, puis celle des grands bouleversements sociaux des années 2000. Zona sur peut ainsi se voir comme un miroir inversé dans lequel se reflètent ces évolutions, la libération des mœurs, la persistance de modèles pourtant dépassés, la fin de la discrimination sociale, la fin d'une vie privilégiée symbolisée par cette maison et son jardin paradisiaque, vouée en fin de compte à la destruction mais à une reconstruction qui n'est pas que symbolique, celle d'un immeuble dans lequel la comadre de Carola compte héberger sa famille. Juan Carlos Valdidia continue de porter son regard sur la réalité bolivienne dans son dernier film, Yvy Maraey (2013), en prenant cette fois lui-même le rôle central, celui d'un réalisateur qui regarde l'autre, ici le guarani, afin de comprendre comment cet autre a contribué à construire une identité, celle que les jailones de Zona sur sont prêts à quitter puisque, pour reprendre les mots de la tante Rosario, « il n'y a rien à faire ici ».

\section{NOTES}

1. Le film Jonás y la ballena rosada est adapté du roman homonyme de Wolfango Montes Vanucci (1987) et le film American visa du roman également homonyme de Juan de Recacoechea (1994).

2. Alors que la tante Rosario et Carola se promènent toutes deux dans le jardin, Carola affirme qu'elle ne veut plus de mari et que lorsqu'elle sera vieille elle finira avec « son » Wilson, l'amour de sa vie comme le lui dit Rosario (63'). Wilson incarne ainsi en quelque sorte l'inversion des valeurs machistes dominantes ; il est la figure de l'homme soumis, relégué à la cuisine, aux tâches subalternes et s'il partage l'espace de l'intimité par excellence de Carola, la salle de bains, c'est de façon clandestine.

3. Olivares Juan José, “Zona sur es un 'retrato de la clase alta de La Paz', Bolivia : Juan Carlos Valdivia" in La Jornada, 05/09/2011. Ressource électronique : [http:// www.jornada.unam.mx/2011/09/05/espectaculos/a14n2esp] (dernière consultation : 26/11/2017). 
4. Voir blog «Literatura en Bolivia », 24 octobre 2013. Ressource électronique: [http:// letrasbol.blogspot.fr/2013/10/cual-es-el-bolivianismo-que-mas-nos.html], (dernière consultation : 26/11/2017).

5. Cf. Pressbook, DVD Zona sur.

6. Sloterdijk Peter, Bulles. Sphères I, Paris, Fayard/Pluriel, 2011 ; Globes. Sphères II, Paris, Fayard/ Pluriel, 2001 ; Écumes. Sphères III, Paris, Fayard/Pluriel, 2013.

7. Cf. Pressbook, DVD Zona sur.

8. Éric Rohmer, L'organisation de l'espace dans le Faust de Murnau, Paris, Ed. Cahiers du cinéma, 2000 (1972).

9. Il est intéressant de souligner à cet égard que le jardin est un espace essentiellement féminin ; en effet, outre que Marcelina en est la jardinière, c'est dans cette sorte de paradis isolé des regards des hommes que se retrouvent Erika et Bernarda pour vivre leur relation amoureuse (plan $\left.n^{\circ} 6\right)$.

10. MORALES ESCOFFIER Sebastián Jorge, La concepción de espacio en el film Zona sur de Juan Carlos Valdivia: una interpretación filosófica, mémoire de fin de diplôme dirigé par Gonzalo Tinajeros, La Paz, Universidad Mayor San Andrés, 2013, p. 85. Nous remercions ici Juan Carlos Valdivia de nous avoir donné accès à ce travail.

11. Molina Mary Carmen, "Ver y no mostrar" in Cinemascine. Ressource électronique : [http:// www.cinemascine.net/criticas/critica/Zona-Sur-ver-y-no-mostrar] (dernière consultation : 26/11/2017).

12. Cf. Pressbook, DVD Zona sur.

13. Voir plans $n^{\circ} 11$ (Wilson), $n^{\circ} 22$ (Wilson), $n^{\circ} 25$ (Bernarda), $n^{\circ} 53$ (Carola), $n^{\circ} 57$ (Patricio), $n$ - 63 (Carola, Patricio, Bernarda, Wilson, Marcelina).

\section{RÉSUMÉS}

Depuis la fin du $\mathrm{XX}^{\mathrm{e}}$ siècle la Bolivie connaît un rééquilibrage social avec l'arrivée d'Evo Morales à la présidence de la république en 2005. La ville de La Paz était le reflet de ce déséquilibre, les plus pauvres occupant les hauteurs de la ville alors que les classes moyennes et supérieures avaient investi les zones de basse altitude. Ces beaux quartiers se sont considérablement étendus et métissés et on les désigne sous le nom de Zona sur. Tel est le titre du quatrième film de Juan Carlos Valdivia, tourné avec une caméra numérique mais distribué en $35 \mathrm{~mm}$ et sorti en 2009. Zona sur dresse le portrait d'une famille issue de cette classe aisée, mais d'une classe aisée en perte de vitesse et en perte de repères. La caméra de Valdivia, constamment en mouvement, circule dans l'espace sans jamais s'attarder sur les personnages. Les regards, les images qui se démultiplient par la prolifération de miroirs, de fenêtres, etc. ne reflètent pas le réel mais la vision d'une réalité inversée.

Desde finales del siglo XX, con la llegada de Evo Morales a la presidencia (2005), Bolivia conoce un nuevo equilibrio social. La Paz era el reflejo de ese desequilibrio: los más pobres vivían en los barrios altos de la ciudad mientras que las clases medias y altas ocupaban las zonas de menor altura. Esos barrios residenciales, que se conocen hoy como Zona sur, se extendieron considerablemente y al mismo tiempo el mestizaje se fue extendiendo. Zona sur es entonces el título del cuarto largometraje de Juan Carlos Valdivia, rodado con una cámara numérica pero distribuido en el formato $35 \mathrm{~mm}$. Estrenado en 2009, Zona sur es el retrato de una familia 
acomodada, pero de una familia acomodada desorientada y en proceso de decadencia. La cámara de Valdivia, en constante movimiento, circula en el espacio sin detenerse nunca en los personajes. Las miradas, las imágenes que se multiplican con la proliferación de espejos, de ventanas, etc. No reflejan lo real sino la visión de una realidad invertida.

INDEX

Palabras claves : Valvidia (Juan Carlos), Zona Sur, Bolivia, cine, sociedad

Mots-clés : Valdivia (Juan Carlos), Zona Sur, Bolivie, cinéma, société

\section{AUTEUR}

\section{ERICH FISBACH}

Université d'Angers, Laboratoire 3L.AM 